\section{Akute Zystitis}

VON H. S. FÜEßL

Die 24-jährige Studentin aus Australien befindet sich auf Europa-Rucksacktour. Sie hat die letzten beiden Nächte in einer überfüllten Jugendherberge verbracht. Am Morgen spürt sie brennende Schmerzen beim Wasserlassen. Seitdem hat sie ständig Harndrang, obwohl sie kaum etwas getrunken hat. Bei der Miktion entleert sich viel weniger Urin, als es ihrem Harndrang entspricht. Zwar bestehen weder Fieber noch allgemeines Krankheitsgefühl, doch sucht die junge Frau wegen der sehr belästigenden Symptomatik bald eine Nothilfe auf.

\section{Situation und Symptomatik}

Die Symptomatik ist typisch für einen akuten Harnwegsinfekt: Dysurie und Pollakisurie. Gelegentlich kann auch blutiger Urin auftreten und es kann zu Schmerzen im Bereich des Beckens kommen. Fieber und Flankenschmerz gehören jedoch nicht zur Symptomatik eines unkomplizierten Harnwegsinfekts. In diesem Fall müsste man an eine Infektion der oberen Harnwege, z. B. eine Pyelonephritis, denken.

Die akute Zystitis ist bei Frauen sehr häufig: Jede fünfte Frau macht mindestens einmal in ihrem Leben eine Harnwegsinfektion (HWI) durch. Bei Männern ist diese Zahl sehr gering, ausgenommen bei Patienten mit Blasenkatheter. Risikofaktoren sind bei jüngeren Frauen anamnestisch durchgemachte HWI, sexuelle Aktivität („Honeymoon-Zystitis“, „Flitterwochen-Blasenentzündung“), Verwendung von Spermiziden; bei älteren Frauen Diabetes mellitus, Demenz, Inkontinenz und Dezensus des Beckenbodens.

\section{Sofortdiagnostik}

- RR, Puls, Temperatur messen

- Klopfschmerz Nierenlager prüfen

- Beklopfen von Wirbelsäule und Flanken

- Auskultation Abdomen, Peristaltik?

- Perkussion und Palpation der Blasenregion (pralle Blase?)

- Urinteststreifen (ergänzend, nicht obligat)

\section{Therapie}

Bei jüngeren Frauen mit einer akuten unkomplizierten Harnwegsinfektion kann eine empirische Einmaltherapie ausreichen, wobei heute wieder zu einer Dreitagesbehandlung tendiert wird. In Betracht kommen Trimethoprim-Sulfamethoxazol, Chinolone (Norfloxacin, Ofloxacin, Ciprofloxacin). Die subjektiven Beschwerden sollten innerhalb von 48 Stunden verschwinden, sonst ist eine weitergehende Diagnostik notwendig. Eine ausreichende „Durchspülung“ (reichliche Flüs- sigkeitszufuhr) unterstützt die Therapie. Bei Schmerzen kann ein krampflösendes Mittel wie Buscopan ${ }^{\circledR}$ gegeben werden.

Ursache der Harnwegsinfekte ist meist eine bakterielle Infektion über die Harnröhre. Die auslösenden Bakterien stammen zu 70-90\% aus der Familie der Escherichia coli. Der Rest wird durch diverse andere Bakterienarten, darunter Proteus und Pseudomonas aeruginosa, hervorgerufen. Diese Keime sprechen auf die Antibiotikabehandlung schlecht an.

\section{Woran noch denken, was noch tun?}

Tritt diese Symptomatik bei einer sonst gesunden Frau erstmals auf, und ergeben sich anamnestisch einschlägige Risiken wie langes Sitzen oder Liegen auf kaltem Boden oder häufiger Geschlechtsverkehr, so kann auf jede weitere Diagnostik verzichtet werden. Handelt es sich dagegen um einen Mann, ein mehrfaches Rezidiv, oder bestehen Grunderkrankungen wie Diabetes mellitus, Z. n. Katheterismus oder Bestrahlung im Beckenbereich, sollte man als Basisdiagnostik ein Urinsediment, eine bakteriologische Untersuchung und eine Sonografie von Nieren und Harnblase durchführen.

\section{Prophylaxe}

Wichtigste Maßnahmen zur Vorbeugung der Zystitis und damit auch der Komplikationen Pyelitis und Pyelonephritis sind reichliche Flüssigkeitszufuhr, Miktion nach Geschlechtsverkehr und Reinigung des weiblichen Genitales von vorn nach hinten. So werden die Gefahren einer Verschleppung von Bakterien in die Harnblase und der Vermehrung der Bakterien weitgehend vermieden. Grunderkrankungen, die den Harnabfluss behindern oder Infektionen begünstigen (Diabetes mellitus!), sollten ausreichend behandelt werden.

\section{Anschrift des Verfassers:}

Prof. Dr. med. H. S. FüeßI, Internist. Abt., Bezirkskrankenhaus Haar, Postfach 1111, D-85540 Haar, E-Mail: fueess|@krankenhaus-haar.de 